\title{
SUPERCONVERGENCE OF MIXED FINITE ELEMENT APPROXIMATIONS OVER QUADRILATERALS
}

\author{
RICHARD E. EWING, MICHAEL M. LIU, AND JUNPING WANG
}

\begin{abstract}
A superconvergence is established in this article for approximate solutions of second order elliptic equations by mixed finite element methods over quadrilaterals. The superconvergence indicates an accuracy of $\mathcal{O}\left(h^{k+2}\right)$ for the mixed finite element approximation if the Raviart-Thomas or Brezzi-Douglas-Fortin-Marini elements of order $k$ are employed with optimal error estimate of $\mathcal{O}\left(h^{k+1}\right)$. Numerical experiments are presented to illustrate the theoretical result.
\end{abstract}

\section{INTRODUCTION}

We are concerned with approximate solutions for the following system of linear equations:

$$
\mathbf{u}=-A \nabla p, \quad \nabla \cdot \mathbf{u}+c p=f, \quad \text { in } \Omega
$$

subject to the Neumann boundary condition:

$$
\mathbf{u} \cdot \nu=g, \quad \text { on } \Gamma \text {. }
$$

Here $\nabla$ is the gradient operator; $\Omega \subset \mathbf{R}^{2}$ is an open bounded domain with boundary $\Gamma ; \nu$ indicates the outward unit normal vector along $\Gamma ; A=\left(a_{i j}\right)_{2 \times 2}$ is a symmetric and positive definite matrix uniformly in $\Omega$. Mixed finite element methods [6] shall be employed to discretize the system (1.1).

Our attention is focused on finite element partitions of $\Omega$ into convex quadrilaterals. In [18], a quadrilateral mixed element has been introduced and analyzed for second order elliptic problems. The quadrilateral elements were constructed by using local mapping techniques for any stable rectangular space such as the Raviart-Thomas [15], BDFM [4], and $D W[10]$ spaces. Details can be found in [18] or Section 2.

The objective of this article is to investigate superconvergence phenomena for the approximate solution of (1.1). Such a study is important in applications to mathematical modeling of fluid flow in porous media since the modeling process requires the determination of a very accurate velocity. If there are places (points or lines) where the approximate solution is more closer to the exact solution than what was predicted by the optimal global error estimate, it would be advantageous to make use of those points or lines in the modeling process.

Various superconvergence results $[9,12,11,17]$ have been established for the mixed finite element approximations of (1.1) on regular rectangular elements. This article shows that

1991 Mathematics Subject Classification. 65N30.

Key words and phrases. Superconvergence, mixed finite element method.

(C) 0000 American Mathematical Society $0000-0000 / 00 \$ 1.00+\$ .25$ per page 
similar superconvergence holds true when quadrilateral elements [18] are employed in the finite element method.

The paper is organized as follows. In $\S 2$, we review the construction of mixed elements over quadrilaterals. In $\S 3$, we present a general framework for convergence/superconvergence estimate. In $\S 4$, we state the main result of this paper. In $\S 5$, we show all the details in the superconvergence analysis. Finally, we present some numerical results in $\S 6$.

\section{MiXed Methods OVER QUADRILATERALS}

Similar to the Galerkin method, the mixed method over quadrilaterals is defined by using mapping techniques to the reference element $\widehat{e}=[-1,1] \times[-1,1]$. In the following we sketch the procedure presented in [18] for the Raviart-Thomas element of order $k(k \geq 0)$.

2.1. Local elements. Let $e$ be any quadrilateral depicted as in Figure 1, where $p_{i}$ stands for the coordinates of the corresponding vertex. The affine map which takes $\widehat{e}$ onto $e$ is given by

$$
\widehat{F}_{e}:\left[\begin{array}{l}
\widehat{x} \\
\widehat{y}
\end{array}\right] \rightarrow S\left[\begin{array}{l}
\widehat{x} \\
\widehat{y}
\end{array}\right]+\mathbf{r}(\widehat{x}+1)(\widehat{y}+1)+\frac{1}{2}\left(p_{2}+p_{4}\right),
$$

where $\mathbf{r}=\frac{1}{4}\left(p_{1}-p_{2}+p_{3}-p_{4}\right)$ and $S=\left(s_{i j}\right)_{2 \times 2}$ is a matrix with $\frac{1}{2}\left(p_{2}-p_{1}\right)$ being the first column and $\frac{1}{2}\left(p_{4}-p_{1}\right)$ the second column.

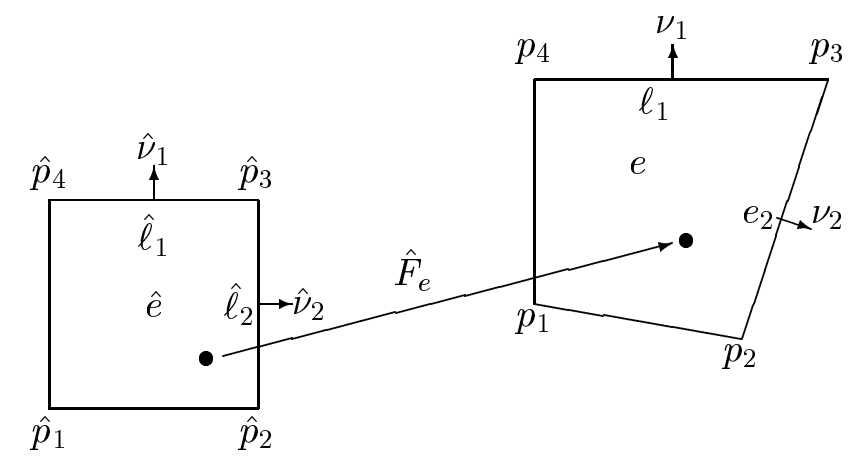

Figure 1. Quadrilateral $e$ and the reference element $\hat{e}$.

Let $G$ be the Jacobi matrix (derivative) of $\widehat{F}_{e}$ and $M=|\operatorname{det}(G)|^{-1} G$. The elementary calculus shows that

$$
G=S+\left[\begin{array}{ll}
r_{1}(\widehat{y}+1) & r_{1}(\widehat{x}+1) \\
r_{2}(\widehat{y}+1) & r_{2}(\widehat{x}+1)
\end{array}\right]
$$

Let $\widehat{V}_{k}(\widehat{e}) \times \widehat{W}_{k}(\widehat{e})$ be the standard Raviart-Thomas rectangular space on the reference element $\hat{e}$ of order $k$. Recall that

$$
\begin{aligned}
\widehat{V}_{k}(\widehat{e}) & =Q_{k+1, k}(\widehat{e}) \times Q_{k, k+1}(\widehat{e}) \\
\widehat{W}_{k}(\widehat{e}) & =Q_{k, k}(\widehat{e}),
\end{aligned}
$$

where $Q_{m, n}(\widehat{e})$ indicates the space of polynomials of degree no more than $m$ and $n$ in $x$ and $y$, respectively. On arbitrary convex quadrilateral $e$, the local Raviart-Thomas space is 
defined by

$$
\begin{array}{cc}
V_{k}(e)=\left\{\mathbf{q}=M \tilde{\mathbf{q}} \circ \widehat{F}_{e}^{-1}:\right. & \left.\tilde{\mathbf{q}} \in \widehat{V}_{k}(\widehat{e})\right\} \\
W_{k}(e)=\left\{w=\widehat{w} \circ \widehat{F}_{e}^{-1}:\right. & \left.\widehat{w} \in \widehat{W}_{k}(\widehat{e})\right\} .
\end{array}
$$

We point out that $V_{k}(e) \subset\left[C^{\infty}(e)\right]^{2}$ and $W_{k}(e) \subset C^{\infty}(e)$ are no longer spaces of polynomials on $e$ unless $e$ is a parallelogram.

2.2. Mixed finite element spaces. Let $\mathcal{T}_{h}$ be a finite element partition of $\Omega$ into triangles and quadrilaterals. On each triangular element $K \in \mathcal{T}_{h}$, the local Raviart-Thomas space for the velocity $\mathbf{u}$ and pressure $p$ are defined in the ordinary way. More precisely, if $P_{k}(e)$ denotes the set of polynomials of total degree no more than $k$, then the pressure space $W_{k}(e)$ is defined by $W_{k}(e)=P_{k}(e)$ and the velocity space is given by

$$
V_{k}(e)=\left\{P_{k}(e) \times P_{k}(e)+\mathbf{x} P_{k}(e)\right\} .
$$

On quadrilateral $e, V_{k}(e)$ and $W_{k}(e)$ are constructed as in (2.2) by using local mappings.

The global finite element space over $\mathcal{T}_{h}$ is defined in the standard way as follows:

$$
\begin{gathered}
\mathbf{V}_{h}=\left\{\mathbf{v} \in H(\operatorname{div} ; \Omega) ;\left.\quad \mathbf{v}\right|_{e} \in V_{k}(e), \forall e \in \mathcal{T}_{h}\right\}, \\
W_{h}=\left\{w \in L^{2}(\Omega) ;\left.\quad w\right|_{e} \in W_{k}(e), \forall e \in \mathcal{T}_{h}\right\},
\end{gathered}
$$

where $\mathbf{V}_{h}$ is the velocity space and $W_{h}$ is that for the pressure.

2.3. Discretization. Define the normal trace operator:

$$
\operatorname{tr}(\mathbf{v})=\left.\mathbf{v} \cdot \nu\right|_{\Gamma}, \quad \mathbf{v} \in \mathbf{V}_{h} .
$$

Let $\Lambda_{h}=\operatorname{tr}\left(\mathbf{V}_{h}\right)$ be the image of $\mathbf{V}_{h}$ and $\mathbf{V}_{0 h}=t r^{-1}(0)$ the null space of $t r$. Let $g_{h} \in \Lambda_{h}$ be an approximation of the Neumann boundary value $g$. Usually, $g_{h}$ is chosen as the $L^{2}$ projection of $g$ on $\Lambda_{h}$.

The mixed method for (1.1) seeks $\mathbf{u}_{h} \in \mathbf{V}_{h}$ and $p_{h} \in W_{h}$ satisfying

$$
\begin{aligned}
a\left(\mathbf{u}_{h}, \mathbf{v}\right)-\left(\nabla \cdot \mathbf{v}, p_{h}\right) & =0, & & \forall \mathbf{v}_{h} \in \mathbf{V}_{0 h}, \\
\left(\nabla \cdot \mathbf{u}_{h}, w\right)+\left(c p_{h}, w\right) & =(f, w), & & \forall w \in W_{h},
\end{aligned}
$$

and the following boundary condition:

$$
\mathbf{u}_{h} \cdot \nu=g_{h}, \quad \text { on } \Gamma .
$$

Here $a(\cdot, \cdot)$ is a bilinear form defined by

$$
a(\mathbf{u}, \mathbf{v})=\int_{\Omega} A^{-1} \mathbf{u} \cdot \mathbf{v} d \Omega
$$

and $(\cdot, \cdot)$ denotes the standard $L^{2}$-inner product. The following error equation holds true:

$$
\begin{aligned}
a\left(\mathbf{u}-\mathbf{u}_{h}, \mathbf{v}\right)-\left(\nabla \cdot \mathbf{v}, p-p_{h}\right) & =0, & \forall \mathbf{v}_{h} \in \mathbf{V}_{0 h} \\
\left(\nabla \cdot\left(\mathbf{u}-\mathbf{u}_{h}\right), w\right)+\left(c\left(p-p_{h}\right), w\right) & =0, & \forall w \in W_{h}
\end{aligned}
$$




\section{A GENERAL FRAMEWORK FOR SUPERCONVERGENCE}

There are two major steps in the general superconvergence analysis. First, one compares the finite element approximation with an appropriately-chosen interpolation of the exact solution in the finite element space. This difference is often far smaller than the global optimal error estimate. Second, one investigates the relation between the exact solution and its interpolation. The objective here is to find special points where the interpolant super-approximates the exact solution. The interpolant is usually locally-defined so that the second step is easy to be carried out.

For the mixed method, the interpolant for the pressure unknown is given locally as follows. Let $\widehat{Q}_{k}$ be the standard $L^{2}$ projection onto the reference pressure space $\widehat{W}_{k}(\widehat{e})$. Then, the projection $Q_{h} w$ for any $w \in L^{2}(\Omega)$ is defined by

$$
Q_{h} w=\widehat{Q}_{k} \widehat{w} \circ \widehat{F}_{e}^{-1}, \quad \text { on } e .
$$

The interpolant for the velocity unknown $\mathbf{u}$, denoted by $\pi \mathbf{u}$, is locally defined to satisfy

$$
\int_{e} \nabla \cdot(\mathbf{u}-\pi \mathbf{u}) w d e=0, \quad \forall w \in W_{k}(e) .
$$

On the Neumann boundary, one should have

$$
\operatorname{tr}(\pi \mathbf{u})=g_{h}
$$

Details on the construction of the operator $\pi$ for various finite element spaces can be found from [6] and [18] on quadrilateral elements.

Theorem 3.1. Let $\left(\mathbf{u}_{h} ; p_{h}\right)$ be the solution of the finite element approximate problem (2.4) and (2.5). Let

$$
\xi=\mathbf{u}_{h}-\pi \mathbf{u}, \quad \eta=p_{h}-Q_{h} p
$$

be the error between the finite element approximation and the projections. Then, there exists a constant $C$ such that

$$
\|\xi\|_{H(\text { div })}+\|\eta\|_{0} \leq C\left(\sup _{\mathbf{v} \in \mathbf{V}_{0 h}} \frac{|a(\mathbf{u}-\pi \mathbf{u}, \mathbf{v})|}{\|\mathbf{v}\|_{H(d i v)}}+\sup _{w \in W_{h}} \frac{\left(c\left(p-Q_{h} p\right), w\right)}{\|w\|_{0}}\right) .
$$

Proof. The proof is basically an application of the known inf-sup condition. In fact, we have from the error equation (2.6) that

and

$$
a(\xi, \mathbf{v})-(\nabla \cdot \mathbf{v}, \eta)=a(\mathbf{u}-\pi \mathbf{u}, \mathbf{v})-\left(\nabla \cdot \mathbf{v}, p-Q_{h} p\right), \quad \forall \mathbf{v} \in \mathbf{V}_{0 h}
$$

Observe that the projections $\pi \mathbf{u}$ and $Q_{h} p$ satisfy

$$
\begin{array}{cc}
(\nabla \cdot(\mathbf{u}-\pi \mathbf{u}), w)=0, & \forall w \in W_{h}, \\
\left(\nabla \cdot \mathbf{v}, p-Q_{h} p\right)=0, & \forall \mathbf{v} \in \mathbf{V}_{0 h} .
\end{array}
$$

It follows from (3.4) and (3.5) that

$$
\begin{aligned}
& a(\xi, \mathbf{v})-(\nabla \cdot \mathbf{v}, \eta)=a(\mathbf{u}-\pi \mathbf{u}, \mathbf{v}), \quad \forall \mathbf{v} \in \mathbf{V}_{0 h} \\
& (\nabla \cdot \xi, w)+(c \eta, w)=\left(c\left(p-Q_{h} p\right), w\right), \quad \forall w \in W_{h},
\end{aligned}
$$

which implies (3.3) by using the standard stability argument [6]. 


\section{MAIN RESULTS}

A quadrilateral partition $\mathcal{T}_{h}$ is said to be uniform if all the elements of $\mathcal{T}_{h}$ are parallelograms of same shape and size. $\mathcal{T}_{h}$ is said to be $h^{2}$-uniform if there exists an absolute constant $C$ such that (a) each element $e \in \mathcal{T}_{h}$ is an $h^{2}$-parallelogram in the sense that $\left\|p_{1}-p_{2}+p_{3}-p_{4}\right\| \leq C h^{2}$, and (b) any two adjacent quadrilaterals (see Figure 2) form an $h^{2}$-parallelogram in the sense that $\left\|p_{4}+p_{1}^{\prime}-2 p_{3}\right\| \leq C h^{2}$.

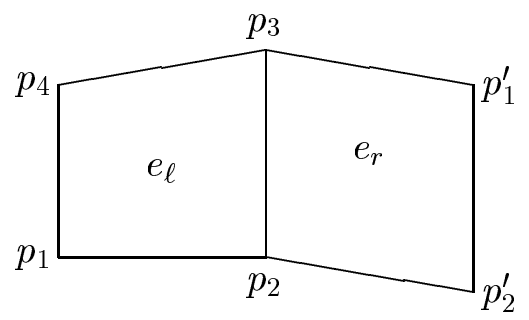

FiguRE 2. Two adjacent quadrilaterals in $\mathcal{T}_{h}$.

Let $D$ be any open bounded domain in $\mathbf{R}^{2}$. For any non-zero integer $m \geq 0$, denote by $H^{m}(D)$ the standard Sobolev space equipped with the following norm:

$$
\|\phi\|_{m, D}=\left(\sum_{|\alpha| \leq m} \int_{D}\left|\partial^{\alpha} \phi\right|^{2} d D\right)^{1 / 2}
$$

where $\alpha=\left(\alpha_{1}, \alpha_{2}\right) \in Z_{+} \times Z_{+},|\alpha|=\alpha_{1}+\alpha_{2}$, and $\partial^{\alpha}=\partial_{x}^{\alpha_{1}} \partial_{y}^{\alpha_{2}}$. For any integer $n \geq 0$, there is also a semi-norm $|\phi|_{n, D}$ defined by

$$
|\phi|_{n, D}=\left(\sum_{|\alpha|=n} \int_{D}\left|\partial^{\alpha} \phi\right|^{2} d D\right)^{1 / 2} .
$$

In the case $D=\Omega$, we shall simplify the notation by $\|\cdot\|_{m} \equiv\|\cdot\|_{m, D}$.

Theorem 4.1. Assume that the finite element partition $\mathcal{T}_{h}$ is $h^{2}$-uniform and $\left(\mathbf{u}_{h} ; p_{h}\right)$ is the approximate solution of (1.1) by using quadrilateral elements of Raviart-Thomas or $B D F M$ of order $k$. If the exact solution $\mathbf{u}$ and $p$ satisfies

$$
\mathbf{u} \in\left[H^{k+2}(\Omega)\right]^{2}, \quad p \in H^{k+1}(\Omega),
$$

then

$$
\left\|\mathbf{u}_{h}-\pi \mathbf{u}\right\|_{H(d i v)}+\left\|Q_{h} p-p_{h}\right\|_{0} \leq C h^{k+2}\left(\|\mathbf{u}\|_{k+2}+\|p\|_{k+1}\right) .
$$

Proof. From Theorem 3.1, we see that it is sufficient to estimate the two terms on the right-hand side of (3.3). The second term can be handled by splitting the integral

$$
\left|\left(c\left(p-Q_{h} p\right), w\right)\right|=\left|\sum_{e \in \mathcal{T}_{h}} \int_{e} c\left(p-Q_{h} p\right) w d x d y\right| .
$$


And on each element $e$, we have

$$
\int_{e} c\left(p-Q_{h} p\right) w d x d y=\int_{e} c\left(p-Q_{h} p\right) w \hat{J}(x, y) \hat{J}^{-1}(x, y) d x d y
$$

where $\hat{J}(x, y)=\left|\frac{\partial(\hat{x}, \hat{y})}{\partial(x, y)}\right|$ is the absolute value of the Jacobian determinant for the mapping $\widehat{F}_{e}^{-1}$. Observe that

$$
\int_{e} \hat{J}(x, y)\left(p-Q_{h} p\right) w=0, \quad \forall w \in W_{h}
$$

Thus, for any real number $\alpha$

$$
\int_{e} c\left(p-Q_{h} p\right) w d x d y=\int_{e}\left(c \hat{J}^{-1}(x, y)-\alpha\right)\left(p-Q_{h} p\right) w \hat{J}(x, y) d x d y .
$$

In particular, if $\alpha$ is the average of $c \hat{J}^{-1}(x, y)$ on the element $e$, then there exists a constant $C$ such that

$$
\left|\int_{e} c\left(p-Q_{h} p\right) w d x d y\right| \leq C h\left\|c \hat{J}^{-1}(x, y)\right\|_{1, \infty} \int_{e}\left|\left(q-Q_{h} p\right) w\right| \hat{J}(x, y) d x d y .
$$

The above inequality can be further estimated as follows:

$$
\left|\int_{e} c\left(p-Q_{h} p\right) w d x d y\right| \leq C h^{k+2}\|c\|_{1, \infty}\|q\|_{k+1, e}\|w\|_{0, e}
$$

Substituting the above into (4.2) yields

$$
\left|\left(c\left(p-Q_{h} p\right), w\right)\right| \leq C h^{k+2}\|c\|_{1, \infty}\|p\|_{k+1}\|w\|_{0} .
$$

This completes the treatment of the second term on the right-hand side of (3.3). The estimate for the first term on the right-hand side of (3.3) is a bitter more complicated, and will be presented in the next section.

\section{Some technical estimates}

The objective of this section is to provide an estimate for $a(\mathbf{u}-\pi \mathbf{u}, \mathbf{v})$ useful in the superconvergence analysis. The estimate will be derived for the Raviat-Thomas element of order $k \geq 0$. The same argument can be carried over to the BDFM elements with a minor modification in the analysis.

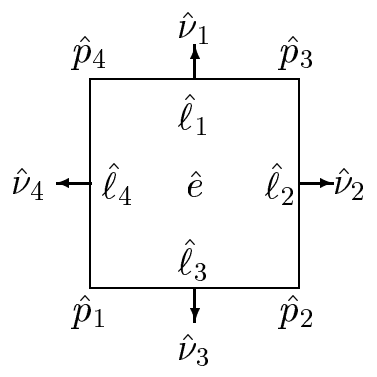

Figure 3 . The reference element $\hat{e}$. 
Let $\tilde{\mathbf{u}}$ be a vector-valued function on the reference element $\hat{e}$. Recall that the interpolation $\hat{\pi} \tilde{\mathbf{u}}$ over $\hat{e}$ is defined by the following linear system:

$$
\begin{gathered}
\int_{\hat{\ell}_{i}}(\tilde{\mathbf{u}}-\hat{\pi} \tilde{\mathbf{u}}) \cdot \hat{\nu}_{i} \phi d s=0, \quad \forall \phi \in P_{k}\left(\hat{\ell}_{i}\right), i=1,2,3,4, \\
\int_{\hat{e}}(\tilde{\mathbf{u}}-\hat{\pi} \tilde{\mathbf{u}}) \cdot \mathbf{v}=0, \quad \forall \mathbf{v} \in Q_{k-1, k} \times Q_{k, k-1} .
\end{gathered}
$$

If $e \in \mathcal{T}_{h}$ is an arbitrary quadrilateral element and $\mathbf{u}$ is a vector-valued function defined on $e$, then the interpolation $\pi \mathbf{u}$ on $e$ is defined by

$$
\pi \mathbf{u}=M\left(\hat{\pi}\left(M^{-1} \hat{\mathbf{u}}\right)\right),
$$

where $\hat{\mathbf{u}}=\mathbf{u} \circ \hat{F}_{e}$ and $\hat{F}_{e}$ is the affine map from $\hat{e}$ to $e$ as defined in Section 2. Details on the property of the projection operator $\pi$ can be found from [18].

Theorem 5.1. If the finite element partition $\mathcal{T}_{h}$ is $h^{2}$-uniform and $\pi \mathbf{u}$ is the interpolation of $\mathbf{u}$ defined as in (5.2), then there exists a constant $C$ such that

$$
|a(\mathbf{u}-\pi \mathbf{u}, \mathbf{v})| \leq C h^{k+2}\left(\|\mathbf{u}\|_{k+2}\|\mathbf{v}\|_{0}+\|\mathbf{u}\|_{k+1}\|\nabla \cdot \mathbf{v}\|_{0}\right), \quad \mathbf{v} \in \mathbf{V}_{0 h} .
$$

Consequently, the first term on the right-hand side of (3.3) is bounded by $C h^{k+2}\|\mathbf{u}\|_{k+2}$ and the superconvergence estimate (4.1) holds true.

The proof of Theorem 5.1 involves a lot of tedious computation and cancellations among quadrilaterals. Interested readers are encouraged to follow the details below.

First we split the integral as follows:

$$
a(\mathbf{u}-\pi \mathbf{u}, \mathbf{v})=\sum_{e \in \mathcal{T}_{h}} \int_{e} A^{-1}(\mathbf{u}-\pi \mathbf{u}) \cdot \mathbf{v} d e .
$$

There are two key steps in the derivation of (5.3). In the first step, we shall investigate some integrals over the reference element $\hat{e}$ which are related to the bilinear form $a(\cdot, \cdot)$. A careful expansion for the integrals will be established. In the second step, we make use of the results established in the first step by transforming the element integrals in (5.4) into integrals over the reference element.

The following two lemmas will accomplish the goal set in the first step.

Lemma 5.1. Let $\tilde{\mathbf{u}}$ be a smooth vector-valued function defined on the reference element $\hat{e}$ and $\tilde{\mathbf{v}}$ be any vector-valued polynomial in $\widehat{V}_{k}(\hat{e})$. Then, there exists a constant $C$ such that

$$
\left|(\tilde{\mathbf{u}}-\hat{\pi} \tilde{\mathbf{u}}, \tilde{\mathbf{v}})_{\hat{e}}\right| \leq C|\tilde{\mathbf{u}}|_{k+2, \hat{e}}\|\tilde{\mathbf{v}}\|_{0, \hat{e}}
$$

Proof. First we observe that if $E(t)=t^{2}-1$, then for any positive integer $m$

$$
\frac{m !}{(2(m+1)) !} \partial_{t}^{m+2} E^{m+1}(t)-t^{m} \in P_{m-2}(t)
$$

is a polynomial of order no more than $m-2$. Note that

$$
(\tilde{\mathbf{u}}-\hat{\pi} \tilde{\mathbf{u}}, \tilde{\mathbf{v}})_{\hat{e}}=\left(\tilde{u}_{1}-\hat{\pi}_{1} \tilde{u}_{1}, \tilde{v}_{1}\right)_{\hat{e}}+\left(\tilde{u}_{2}-\hat{\pi}_{2} \tilde{u}_{2}, \tilde{v}_{2}\right)_{\hat{e}}
$$

We shall present an analysis for the first term $\left(\tilde{u}_{1}-\hat{\pi}_{1} \tilde{u}_{1}, \tilde{v}_{1}\right)_{\hat{e}}$ only; the second term can be analyzed similarly. 
From the Taylor expansion and the fact $\tilde{v}_{1} \in Q_{k+1, k}$ we see that

$$
\tilde{v}_{1}-\frac{1}{k !} x^{k} \partial_{\hat{x}}^{k} \tilde{v}_{1}(0, \hat{y})-\frac{1}{(k+1) !} x^{k+1} \partial_{\hat{x}}^{k+1} \tilde{v}_{1}(0, \hat{y}) \in Q_{k-1, k} .
$$

This together with (5.6) implies

$$
\tilde{v}_{1}-\frac{1}{(2(k+1)) !} \partial_{\hat{x}}^{k+2} E^{k+1}(\hat{x}) \partial_{\hat{x}}^{k} \tilde{v}_{1}(0, \hat{y})-\frac{1}{(2(k+2)) !} \partial_{\hat{x}}^{k+3} E^{k+2}(\hat{x}) \partial_{\hat{x}}^{k+1} \tilde{v}_{1}(0, \hat{y}) \in Q_{k-1, k} .
$$

Therefore, the definition of $\hat{\pi} \tilde{\mathbf{u}}$ leads to the following

$$
\begin{aligned}
\left(\tilde{u}_{1}-\hat{\pi}_{1} \tilde{u}_{1}, \tilde{v}_{1}\right)_{\hat{e}}= & \frac{1}{(2(k+1)) !} \int_{\hat{e}}\left(\tilde{u}_{1}-\hat{\pi}_{1} \tilde{u}_{1}\right) \partial_{\hat{x}}^{k+2} E^{k+1}(\hat{x}) \partial_{\hat{x}}^{k} \tilde{v}_{1}(0, \hat{y}) \\
& +\frac{1}{(2(k+2)) !} \int_{\hat{e}}\left(\tilde{u}_{1}-\hat{\pi}_{1} \tilde{u}_{1}\right) \partial_{\hat{x}}^{k+3} E^{k+2}(\hat{x}) \partial_{\hat{x}}^{k+1} \tilde{v}_{1}(0, \hat{y}) \\
= & \frac{1}{(2(k+1)) !} I_{1}+\frac{1}{(2(k+2)) !} I_{2} .
\end{aligned}
$$

where $I_{j}$ are defined accordingly.

On the edges $\hat{\ell}_{2}$ and $\hat{\ell}_{4}, \partial_{\hat{x}}^{k} \tilde{v}_{1}(0, \hat{y}) \in P_{k}(\hat{y})$ and $\partial_{i} E^{m}(\hat{x})=0$ for all $i<m$. Thus, we have from the integration by parts and the first equation of (5.1) that

$$
\begin{aligned}
I_{1}= & (-1)^{k+2} \int_{\hat{e}} E^{k+1}(\hat{x}) \partial_{\hat{x}}^{k+2} \tilde{u}_{1} \partial_{\hat{x}}^{k} \tilde{v}_{1}(0, \hat{y}) \\
& +\left(\int_{\hat{\ell}_{2}}-\int_{\hat{\ell}_{4}}\right)\left(\tilde{u}_{1}-\hat{\pi}_{1} \tilde{u}_{1}\right) \partial_{\hat{x}}^{k+1} E^{k+1}(\hat{x}) \partial_{\hat{x}}^{k} \tilde{v}_{1}(0, \hat{y}) \\
= & (-1)^{k+2} \int_{\hat{e}} E^{k+1}(\hat{x}) \partial_{\hat{x}}^{k+2} \tilde{u}_{1} \partial_{\hat{x}}^{k} \tilde{v}_{1}(0, \hat{y}) \\
\leq & C\left|\tilde{u}_{1}\right|_{k+2, \hat{e}}\left|\tilde{v}_{1}(0, \hat{y})\right|_{k, \hat{e}} \leq C\left|\tilde{u}_{1}\right|_{k+2, \hat{e}} \mid \tilde{v}_{1} \|_{0, \hat{e}},
\end{aligned}
$$

where we have used the standard inverse inequality for finite element functions in the last line. The same argument can be applied to the term $I_{2}$, yielding

$$
I_{2}=(-1)^{k+2} \int_{\hat{e}} \partial_{\hat{x}} E^{k+2}(\hat{x}) \partial_{\hat{x}}^{k+2} \tilde{u}_{1} \partial_{\hat{x}}^{k+1} \tilde{v}_{1}(0, \hat{y}) \leq C\left|\tilde{u}_{1}\right|_{k+2, \hat{e}}\left\|\tilde{v}_{1}\right\|_{0, \hat{e}}
$$

Substituting (5.9) and (5.10) into (5.8) yields the estimate (5.5).

Lemma 5.2. Let $\tilde{\mathbf{u}}$ be a smooth vector-valued function defined on the reference element $\hat{e}$ and $\tilde{\mathbf{v}}$ be any vector-valued polynomial in $\widehat{V}_{k}(\hat{e})$. Then,

$$
\left(\tilde{u}_{1}-\hat{\pi}_{1} \tilde{u}_{1}, v_{2}\right)_{\hat{e}}=R+\left(\int_{\hat{\ell}_{4}}-\int_{\hat{\ell}_{2}}\right) \partial_{\hat{y}}^{k+1} \tilde{u}_{1} E^{k+1}(\hat{y}) \partial_{\hat{y}}^{k} \tilde{v}_{1} d \hat{y}
$$

where $R=R\left(\tilde{u}_{1}, \tilde{\mathbf{v}}\right)$ is a quantity with the following estimate:

$$
|R| \leq C\left(\left|\tilde{u}_{1}\right|_{k+2, \hat{e}}\|\tilde{\mathbf{v}}\|_{0, \hat{e}}+\left|\tilde{u}_{1}\right|_{k+1, \hat{e}}\|\hat{\nabla} \cdot \tilde{\mathbf{v}}\|_{0, \hat{e}}\right) .
$$

Proof. Since $\tilde{v}_{2} \in Q_{k, k+1}$, then $\tilde{v}_{2}-\frac{1}{(k+1) !} y^{k+1} \partial_{\hat{y}}^{k+1} \tilde{v}_{2}(\hat{x}, \hat{y}) \in Q_{k+1, k}$. It follows from (5.6) that

$$
\tilde{v}_{2}^{\prime}=\tilde{v}_{2}-\frac{1}{(2(k+1)) !} \partial_{\hat{y}}^{k+1}\left(E^{k+1}(\hat{y})\right) \partial_{\hat{y}}^{k+1} \tilde{v}_{2}(\hat{x}, \hat{y}) \in Q_{k+1, k}
$$


Thus, Lemma 5.1 can be applied to yield

$$
\left|\left(\tilde{u}_{1}-\hat{\pi}_{1} \tilde{u}_{1}, \tilde{v}_{2}^{\prime}\right)_{\hat{e}}\right| \leq C\left|\tilde{u}_{1}\right|_{k+2, \hat{e}}|| \tilde{v}_{2}^{\prime}\left\|_{0, \hat{e}} \leq C\left|\tilde{u}_{1}\right|_{k+2, \hat{e}}|| \tilde{v}_{2}\right\|_{0, \hat{e}} .
$$

Since

$$
\left(\tilde{u}_{1}-\hat{\pi}_{1} \tilde{u}_{1}, \tilde{v}_{2}\right)_{\hat{e}}=\left(\tilde{u}_{1}-\hat{\pi}_{1} \tilde{u}_{1}, \tilde{v}_{2}^{\prime}\right)_{\hat{e}}+\left(\tilde{u}_{1}-\hat{\pi}_{1} \tilde{u}_{1}, \tilde{v}_{2}-\tilde{v}_{2}^{\prime}\right)_{\hat{e}}
$$

and

$$
\begin{aligned}
& (2 k+2) !\left(\tilde{u}_{1}-\hat{\pi}_{1} \tilde{u}_{1}, \tilde{v}_{2}-\tilde{v}_{2}^{\prime}\right) \hat{e}=\left(\tilde{u}_{1}-\hat{\pi}_{1} \tilde{u}_{1}, \partial_{\hat{y}}^{k+1}\left(E^{k+1}(\hat{y})\right) \partial_{\hat{y}}^{k+1} \tilde{v}_{2}(\hat{x}, \hat{y})\right)_{\hat{e}} \\
& =\int_{\hat{e}} \partial_{\hat{y}}^{k+1} \tilde{u}_{1} E^{k+1}(\hat{y}) \partial_{\hat{y}}^{k}(\operatorname{div} \tilde{\mathbf{v}})-\int_{\hat{e}} \partial_{\hat{y}}^{k+1} \tilde{u}_{1} E^{k+1}(\hat{y}) \partial_{\hat{y}}^{k}\left(\partial_{\hat{x}} \tilde{v}_{1}\right) \\
& =\int_{\hat{e}} \partial_{\hat{y}}^{k+1} \tilde{u}_{1} E^{k+1}(\hat{y}) \partial_{\hat{y}}^{k}(\operatorname{div} \tilde{\mathbf{v}})+\int_{\hat{e}} \partial_{\hat{x}} \partial_{\hat{y}}^{k+1} \tilde{u}_{1} E^{k+1}(\hat{y}) \partial^{k} \tilde{v}_{1} \\
& \quad+\left(\int_{\hat{\ell}_{4}}-\int_{\hat{\ell}_{2}}\right) \partial_{\hat{y}}^{k+1} \tilde{u}_{1} E^{k+1}(\hat{y}) \partial_{\hat{y}}^{k} \tilde{v}_{1} d \hat{y}
\end{aligned}
$$

by letting

$R=\left(\tilde{u}_{1}-\hat{\pi}_{1} \tilde{u}_{1}, \tilde{v}_{2}^{\prime}\right)_{\hat{e}}+\frac{1}{(2 k+2) !}\left(\int_{\hat{e}} \partial_{\hat{y}}^{k+1} \tilde{u}_{1} E^{k+1}(\hat{y}) \partial_{\hat{y}}^{k}(\operatorname{div} \tilde{\mathbf{v}})+\int_{\hat{e}} \partial_{\hat{x}} \partial_{\hat{y}}^{k+1} \tilde{u}_{1} E^{k+1}(\hat{y}) \partial^{k} \tilde{v}_{1}\right)$,

we obtain (5.11). The estimate (5.12) is straightforward from (5.13) and the inverse inequality.

The second step needs the following results:

Lemma 5.3. Let $\mathcal{T}_{h}$ be an $h^{2}$-uniform partition of $\Omega$. There exists a constant $C$ such that for any $e \in \mathcal{T}_{h}$,

$$
\begin{aligned}
\left|\hat{\partial}^{\alpha} \hat{F}_{e}\right| & \leq \begin{cases}C h^{|\alpha|}, & \alpha=(1,0),(0,1), \text { or }(1,1), \\
0, & |\alpha| \geq 2 .\end{cases} \\
\left|\hat{\partial}^{\alpha} M^{-1}\right| & \leq \begin{cases}C h^{|\alpha|+1}, & |\alpha| \leq 1 \\
0, & |\alpha| \geq 2 .\end{cases}
\end{aligned}
$$

A proof for Lemma 5.3 can be given by using the construction of the affine map $\widehat{F}_{e}$ and the matrix $M^{-1}=|\operatorname{det}(G)|^{-1} G$ in Section 2 .

Lemma 5.4. Let e be an $h^{2}$-parallelogram and $\mathbf{u}$ be a smooth vector-valued function defined on e. Let $\hat{F}_{e}$ be the affine map from the reference element to $e$ and

$$
\hat{\mathbf{u}}=\mathbf{u} \circ \hat{F}_{e}, \quad \tilde{\mathbf{u}}=M^{-1} \hat{\mathbf{u}},
$$

where $M$ is defined as in Section 2. Then, there exists a constant $C$ such that for any integer $m \geq 0$,

$$
\begin{gathered}
|\tilde{\mathbf{u}}|_{m, \hat{e}} \leq C h^{m}\|\mathbf{u}\|_{m, e}, \\
|\hat{\nabla} \cdot \tilde{\mathbf{u}}|_{0, \hat{e}} \leq C h\|\nabla \cdot \mathbf{u}\|_{0, e} .
\end{gathered}
$$


Proof. Since $\tilde{\mathbf{u}}=M^{-1} \hat{\mathbf{u}}$, then from the product rule we have

$$
\hat{\partial}^{\alpha} \tilde{\mathbf{u}}=\sum_{\eta \leq \alpha} \hat{\partial}^{\alpha-\eta} \hat{\mathbf{u}} \hat{\partial}^{\eta} M^{-1}=\sum_{|\eta| \leq 1} \hat{\partial}^{\alpha-\eta} \hat{\mathbf{u}} \hat{\partial}^{\eta} M^{-1} .
$$

Using the estimate on $M^{-1}$ in Lemma 5.3, we obtain

$$
|\tilde{\mathbf{u}}|_{m, p, \hat{e}} \leq C\left(h|\hat{\mathbf{u}}|_{m, \hat{e}}+h^{2}|\hat{\mathbf{u}}|_{m-1, \hat{e}}\right)
$$

Since $\hat{\mathbf{u}}=\mathbf{u} \circ \hat{F}_{e}$, then by a change of variable we get

$$
|\hat{\mathbf{u}}|_{j, \hat{e}} \leq C h^{j-1}\|\mathbf{u}\|_{j, e}, \quad j \geq 0
$$

where we have used the first inequality of (5.14). This, together with (5.17), verifies the inequality in (5.15). The estimate in (5.16) is a by-product of the identity

$$
\widehat{\nabla} \cdot \tilde{\mathbf{u}}=|\operatorname{det}(G)| \nabla \cdot \mathbf{u},
$$

which was derived in [18].

The rest of this section is devoted to a proof of Theorem 5.1. From (5.4), it suffices to estimate the integral on each element. To this end, let $\widehat{F}_{e}$ be the affine map from the reference element $\hat{e}$ to $e$ as described in Section 2. For convenience, we order the vertices $\hat{p}_{i}$ in a counterclockwise direction and $p_{i}=\widehat{F}_{e}\left(\hat{p}_{i}\right)$. It follows from substitution of variables that

$$
\int_{e} A^{-1}(\mathbf{u}-\pi \mathbf{u}) \cdot \mathbf{v} d e=\int_{\hat{e}} \widehat{A}^{-1}(\hat{\mathbf{u}}-\widehat{\pi \mathbf{u}}) \cdot \widehat{\mathbf{v}} \operatorname{det}\left(G_{e}\right) d \hat{e},
$$

where $\hat{q}=q \circ \widehat{F}_{e}$ for any (scalar, vector, or matrix valued) function $q=q(x, y)$ on $e$. Let

$$
\tilde{\mathbf{u}}=M^{-1} \hat{\mathbf{u}}, \tilde{\mathbf{v}}=M^{-1} \hat{\mathbf{v}} .
$$

Then, $\hat{\mathbf{u}}=M \tilde{\mathbf{u}}, \hat{\mathbf{v}}=M \tilde{\mathbf{v}}$, and from (5.2)

$$
\widehat{\pi \mathbf{u}}=M(\hat{\pi} \tilde{\mathbf{u}}) .
$$

With $B_{e}=M^{t} \widehat{A}^{-1} M \operatorname{det}\left(G_{e}\right)$, we see that

$$
\int_{e} A^{-1}(\mathbf{u}-\pi \mathbf{u}) \cdot \mathbf{v} d e=\int_{\hat{e}} B_{e}(\tilde{\mathbf{u}}-\hat{\pi} \tilde{\mathbf{u}}) \cdot \tilde{\mathbf{v}} d \hat{e} .
$$

Let $\bar{B}_{e}$ be the average of $B_{e}$ on the reference element $\hat{e}$. Since the quadrilateral $e$ is an $h^{2}$-parallelogram, then

$$
\left|B_{e}-\bar{B}_{e}\right| \leq C h
$$

for some constant $C$. It follows that

$$
\int_{\hat{e}} B_{e}(\tilde{\mathbf{u}}-\hat{\pi} \tilde{\mathbf{u}}) \cdot \tilde{\mathbf{v}} d \hat{e}=\int_{\hat{e}}\left(B_{e}-\bar{B}_{e}\right)(\tilde{\mathbf{u}}-\hat{\pi} \tilde{\mathbf{u}}) \cdot \tilde{\mathbf{v}} d \hat{e}+\int_{\hat{e}} \bar{B}_{e}(\tilde{\mathbf{u}}-\hat{\pi} \tilde{\mathbf{u}}) \cdot \tilde{\mathbf{v}} d \hat{e} .
$$

Since the first term on the right-hand side of the above equality is bounded by

$$
C h\|\tilde{\mathbf{u}}-\hat{\pi} \tilde{\mathbf{u}}\|_{0, \hat{e}}\|\tilde{\mathbf{v}}\|_{0, \hat{e}},
$$

which is further bounded by

$$
C h|\tilde{\mathbf{u}}|_{k+1, \hat{e}}\|\tilde{\mathbf{v}}\|_{0, \hat{e}} \leq C h^{k+2}\|\mathbf{u}\|_{k+1, e}\|\mathbf{v}\|_{0, e},
$$


by summing (5.20) over all the elements $e$ we obtain

$$
|a(\mathbf{u}-\pi \mathbf{u}, \mathbf{v})| \leq C h^{k+2}\|\mathbf{u}\|_{k+1}\|\mathbf{v}\|_{0}+\left|\sum_{e} \int_{\hat{e}} \bar{B}_{e}(\tilde{\mathbf{u}}-\hat{\pi} \tilde{\mathbf{u}}) \cdot \tilde{\mathbf{v}} d \hat{e}\right| .
$$

Therefore, it suffices to estimate the $\operatorname{sum} \sum_{e} \int_{\hat{e}} \bar{B}_{e}(\tilde{\mathbf{u}}-\hat{\pi} \tilde{\mathbf{u}}) \cdot \tilde{\mathbf{v}} d \hat{e}$.

Let $\bar{B}_{e}=\left(\bar{a}_{i j}^{e}\right)_{2 \times 2}$. Then,

$$
\int_{\hat{e}} \bar{B}_{e}(\tilde{\mathbf{u}}-\hat{\pi} \tilde{\mathbf{u}}) \cdot \tilde{\mathbf{v}} d \hat{e}=\sum_{i, j=1}^{2} \bar{a}_{i j}^{e}\left(\tilde{u}_{i}-\hat{\pi}_{i} \tilde{u}_{i}, \tilde{v}_{j}\right)_{\hat{e}}
$$

Now is the time to use the results in Lemmas 5.1 and 5.2. First, we see from Lemma 5.1 that

$$
\left|\left(\tilde{u}_{1}-\hat{\pi}_{1} \tilde{u}_{1}, \tilde{v}_{1}\right)_{\hat{e}}\right|+\left|\left(\tilde{u}_{2}-\hat{\pi}_{2} \tilde{u}_{2}, \tilde{v}_{2}\right)_{\hat{e}}\right| \leq C \mid \tilde{\mathbf{u}}_{\mid k+2, \hat{e}}\|\tilde{\mathbf{v}}\|_{0, \hat{e}}
$$

which gives the expected superconvergence estimate. It remains to deal with the cross terms $\left(\tilde{u}_{1}-\hat{\pi}_{1} \tilde{u}_{1}, \tilde{v}_{2}\right)_{\hat{e}}$ and $\left(\tilde{u}_{2}-\hat{\pi}_{2} \tilde{u}_{2}, \tilde{v}_{1}\right) \hat{e}$. From Lemma 5.2, those cross terms have expansion as illustrated in (5.11) featuring area and line integrals. The area integrals are bounded by

$$
C\left(|\tilde{\mathbf{u}}|_{k+2, \hat{e}}\|\tilde{\mathbf{v}}\|_{0}+|\tilde{\mathbf{u}}|_{k+1, \hat{e}}\|\hat{\nabla} \cdot \tilde{\mathbf{v}}\|_{0}\right),
$$

which gives rise to the required superconvergence by using Lemma 5.4. The line integrals, in general, prevent us from having a superconvergence unless the finite element partition is $h^{2}$-uniform. In fact, if the partition is $h^{2}$-uniform, then there will be a good cancellation among those line integrals.

To see how cancellation works, we use the fact that the derivatives in the line integral are taken in the direction of the line. Thus, the sum of all the line integrals actually looks like the following:

$$
\sum_{e \in T_{h}} \sum_{i \neq j} \bar{a}_{i j}^{e} \sum_{k=1}^{4} \int_{\hat{\ell}_{k}^{e}} \bar{E}(\hat{\tau}) \partial_{\hat{\tau}}^{k+1} \tilde{\mathbf{u}} \cdot \hat{\nu}_{k} \partial_{\hat{\tau}}^{k} \tilde{\mathbf{v}} \cdot \hat{\nu}_{k} d s,
$$

where $\bar{E}(\hat{\tau})$ is a polynomial defined on the interval $[-1,1]$. Recall that on each edge $\hat{\ell}_{k}^{e}$ we have

$$
\tilde{\mathbf{v}} \cdot \hat{\nu}_{k}=M \tilde{\mathbf{v}} \cdot M^{-T} \hat{\nu}_{k}=\left(\left|\ell_{k}^{e}\right| \mathbf{v} \cdot \nu\right) \circ \hat{F}_{e},
$$

where $\ell_{k}^{e}=\hat{F}_{e}\left(\hat{\ell}_{k}\right)$ with length $\left|\ell_{k}^{e}\right|$. Thus, (5.22) can be rewritten as

$$
\sum_{e \in T_{h}} \sum_{i \neq j} \bar{a}_{i j}^{e} \sum_{k=1}^{4}\left|\ell_{k}^{e}\right|^{2 k+2} \int_{\ell_{k}^{e}} \bar{E}(\tau) \partial_{\tau}^{k+1}\left(\mathbf{u} \cdot \nu_{k}\right) \partial_{\tau}^{k}\left(\mathbf{v} \cdot \nu_{k}\right) d s .
$$

If $\ell_{k}^{e}$ happens to be on the boundary of $\Omega$, then the trial function $\mathbf{v}$ has vanishing normal component and the corresponding line integrals disappear. If $\ell_{k}^{e}$ is an interior edge, then there must be a neighboring element, say $e^{\prime}$, of $e$ which shares $\ell_{k}^{e}$ as an edge. Since the normal vectors on $\ell_{k}^{e}$ have opposite direction as seen from $e$ and $e^{\prime}$. It follows that the sum of the line integrals is bounded by

$$
C \sum_{i \neq j} \sum_{\ell \in \Gamma_{h}} \int_{\ell}\left|\bar{a}_{i j}^{e}-\bar{a}_{i j}^{e^{\prime}}\right||\ell|^{2 k+2}\left|\partial_{\tau}^{k+1}(\mathbf{u} \cdot \nu)\right|\left|\partial_{\tau}^{k}(\mathbf{v} \cdot \nu)\right| d \ell,
$$


where $\Gamma_{h}$ is the collection of interior edges. If the finite element partition is $h^{2}$-uniform, then it is not hard to see that

Thus, (5.24) is bounded by

$$
\left|\bar{a}_{i j}^{e}-\bar{a}_{i j}^{e^{\prime}}\right| \leq C h
$$

$$
C h^{2 k+3} \sum_{\ell \in \Gamma_{h}}|\mathbf{u} \cdot \nu|_{k+1, \ell}|\mathbf{v} \cdot \nu|_{k, \ell} \leq C h^{k+3} \sum_{\ell \in \Gamma_{h}}|\mathbf{u} \cdot \nu|_{k+1, \ell}\|\mathbf{v} \cdot \nu\|_{0, \ell}
$$

where we have used the inverse inequality to estimate $|\mathbf{v} \cdot \nu|_{k, \ell}$. Finally, we use the wellknown estimates:

$$
\begin{aligned}
\|\mathbf{v} \cdot \nu\|_{0, \ell} & \leq C h^{-\frac{1}{2}}\|\mathbf{v}\|_{0, e} \\
\|\mathbf{u} \cdot \nu\|_{k+1, \ell} & \leq C h^{-\frac{1}{2}}\|\mathbf{u}\|_{k+2, e}
\end{aligned}
$$

to bound the sum of line integrals by

$$
C h^{k+2} \sum_{e \in \mathcal{T}_{h}}\|\mathbf{u}\|_{k+2, e}\|\mathbf{v}\|_{0, e},
$$

which gives the required superconvergence estimate.

\section{NumericAl RESUlts}

The objective of this section is to confirm the superconvergence result through numerical experiments. The Raviart-Thomas element of lowest order is employed in the computation on various domains. All the examples tested have exact solution $p=\sin (x) \sin (y)$ with $c=1$, and the velocity can be computed by using the first equation of (1.1).

The error between the approximate solution $\left(\mathbf{u}_{h}, p_{h}\right)$ and the projection $\left(\pi \mathbf{u}, Q_{h} p\right)$ is measured in $L^{2}$ and $L^{\infty}$ norms. Note that the theory predicts the following error estimates:

$$
\left\|p_{h}-Q_{h} p\right\|_{0} \leq C h^{\alpha_{1}}, \quad\left\|\mathbf{u}_{h}-\pi \mathbf{u}\right\|_{0} \leq C h^{\alpha_{2}}
$$

with $\alpha_{1}=2$ in all examples tested below. The index $\alpha_{2}$ is mesh and problem dependent. The least-square method will be employed to provide an estimate on the index $\alpha_{i}$.

EXAMPLE 6.1. The domain $\Omega=(0,1) \times(0,1)$ and the coefficient matrix $A$ is chosen as the identity matrix. The Dirichlet boundary condition is considered in the computation, and the result is illustrated in Table 1.

TABLE 1. Numerical results for Example 6.1

\begin{tabular}{|c||c|c|c|c|}
\hline$\#$ levels $J$ & $\left\|\pi \mathbf{u}-\mathbf{u}_{h}\right\|_{0}$ & $\left\|\pi \mathbf{u}-\mathbf{u}_{h}\right\|_{\infty}$ & $\left\|p_{h}-Q_{h} p\right\|_{0}$ & $\left\|p_{h}-Q_{h} p\right\|_{\infty}$ \\
\hline 2 & $1.8956 \mathrm{E}-3$ & $2.3702 \mathrm{E}-3$ & $3.3685 \mathrm{E}-3$ & $7.5147 \mathrm{E}-3$ \\
\hline 3 & $4.7729 \mathrm{E}-4$ & $6.0831 \mathrm{E}-4$ & $8.5246 \mathrm{E}-4$ & $2.1003 \mathrm{E}-3$ \\
\hline 4 & $1.1951 \mathrm{E}-4$ & $1.5315 \mathrm{E}-4$ & $2.1378 \mathrm{E}-4$ & $5.5154 \mathrm{E}-4$ \\
\hline 5 & $3.2523 \mathrm{E}-5$ & $4.4603 \mathrm{E}-5$ & $5.2805 \mathrm{E}-5$ & $1.4106 \mathrm{E}-4$ \\
\hline \hline$\alpha$ & 1.96 & 1.92 & 2.0 & 1.91 \\
\hline
\end{tabular}

The finite element partition $\mathcal{T}_{h}$ is obtained by a sequence of uniform refinements on the coarsest level. For example, the partition $\mathcal{T}_{h}$ corresponding to $J=1$ is given by breaking $\Omega$ into four smaller rectangles by connecting the midpoints of each pair of opposite edges of $\Omega$. It is clear that the mesh size for $\mathcal{T}_{h}$ is $h=2^{-J}$. 
The last row in Table 1 gives estimates for the index $\alpha_{i}$. Note that the result developed in $[9,12,17]$ claims $\alpha_{2}=2$ for this example. This theory is clearly confirmed by the numerical results in Table 1.

EXAMPLE 6.2. Here we consider a quadrilateral domain depicted in Figure 4. The differential equation and the boundary condition are the same as in Example 6.1. The computational results are presented in Table 2.

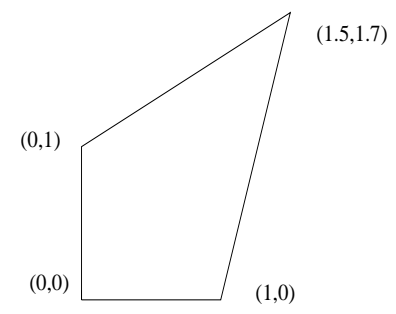

Figure 4. A quadrilateral domain $\Omega$.

TABLE 2. Numerical results for Example 6.2

\begin{tabular}{|c||c|c|c|c|}
\hline$\#$ levels $J$ & $\left\|\pi \mathbf{u}-\mathbf{u}_{h}\right\|_{0}$ & $\left\|\pi \mathbf{u}-\mathbf{u}_{h}\right\|_{\infty}$ & $\left\|p_{h}-Q_{h} p\right\|_{0}$ & $\left\|p_{h}-Q_{h} p\right\|_{\infty}$ \\
\hline 2 & $5.8703 \mathrm{E}-3$ & $8.4529 \mathrm{E}-3$ & $1.1668 \mathrm{E}-2$ & $3.1035 \mathrm{E}-2$ \\
\hline 3 & $1.6445 \mathrm{E}-3$ & $4.8276 \mathrm{E}-3$ & $2.9737 \mathrm{E}-3$ & $8.8569 \mathrm{E}-3$ \\
\hline 4 & $4.4414 \mathrm{E}-4$ & $2.5037 \mathrm{E}-3$ & $7.4782 \mathrm{E}-4$ & $2.3146 \mathrm{E}-3$ \\
\hline 5 & $1.1420 \mathrm{E}-4$ & $1.2497 \mathrm{E}-3$ & $1.8908 \mathrm{E}-4$ & $5.8773 \mathrm{E}-4$ \\
\hline \hline$\alpha$ & 1.89 & 0.92 & 1.98 & 1.91 \\
\hline
\end{tabular}

Note that the superconvergence developed in this paper is not applicable for Dirichlet boundary value problems because the trial function $\mathbf{v}$ does not vanish on the boundary $\Gamma$ in the normal direction. But the argument presented in this paper can be used to yield the following estimate:

$$
\left\|\mathbf{u}_{h}-\pi \mathbf{u}\right\|_{0} \leq C h^{k+1.5} .
$$

In particular, the theory gives a superconvergence with $\alpha_{2}=1.5$ when $k=0$. The numerical estimate on $\alpha_{2}$ for this example is $\alpha_{2}=1.89$ which is clearly better than the theory. No superconvergence is seen for the approximate velocity in the $L^{\infty}$ norm.

Example 6.3. The domain $\Omega$ is shown in Figure 5. The initial partition contains three quadrilaterals. The coefficient matrix is given by

$$
A=\left[\begin{array}{cc}
1+e^{y} & 0.5 \\
0.5 & 1+e^{x}
\end{array}\right]
$$

Again, the Dirichlet boundary condition is used in this test. 


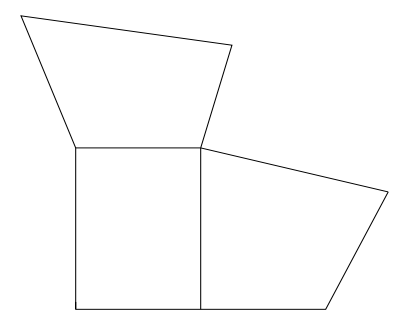

FiguRE 5. An $L$-shaped domain $\Omega$.

TABle 3. Numerical results for Example 6.3

\begin{tabular}{|c||c|c|c|c|}
\hline$\#$ levels $J$ & $\left\|\pi \mathbf{u}-\mathbf{u}_{h}\right\|_{0}$ & $\left\|\pi \mathbf{u}-\mathbf{u}_{h}\right\|_{\infty}$ & $\left\|p_{h}-Q_{h} p\right\|_{0}$ & $\left\|p_{h}-Q_{h} p\right\|_{\infty}$ \\
\hline 2 & $4.6614 \mathrm{E}-2$ & 0.1588 & $1.0438 \mathrm{E}-2$ & $2.4613 \mathrm{E}-2$ \\
\hline 3 & $1.2833 \mathrm{E}-2$ & $5.9276 \mathrm{E}-2$ & $2.6323 \mathrm{E}-3$ & $6.6552 \mathrm{E}-3$ \\
\hline 4 & $3.3909 \mathrm{E}-3$ & $2.6857 \mathrm{E}-2$ & $6.5943 \mathrm{E}-4$ & $1.7161 \mathrm{E}-3$ \\
\hline 5 & $8.8407 \mathrm{E}-4$ & $1.2707 \mathrm{E}-2$ & $1.6496 \mathrm{E}-4$ & $4.3523 \mathrm{E}-4$ \\
\hline \hline$\alpha$ & 1.91 & 1.21 & 2.0 & 1.94 \\
\hline
\end{tabular}

EXAMPLE 6.4. Here the coefficient matrix is the identity $A=I$. The domain $\Omega$ is the disc of radis 2 . The Neumann boundary condition is used in the computation. The initial partition of $\Omega$ comprises five quadrilaterals depicted in Figure 6.

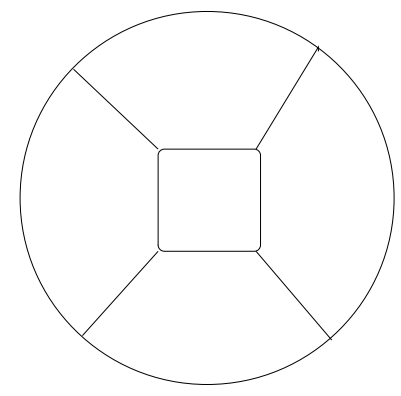

FiguRE 6. An initial partition for a test domain $\Omega$.

TABle 4. Numerical results for Example 6.4

\begin{tabular}{|c||c|c|c|c|}
\hline \# levels $J$ & $\left\|\pi \mathbf{u}-\mathbf{u}_{h}\right\|_{0}$ & $\left\|\pi \mathbf{u}-\mathbf{u}_{h}\right\|_{\infty}$ & $\left\|p_{h}-Q_{h} p\right\|_{0}$ & $\left\|p_{h}-Q_{h} p\right\|_{\infty}$ \\
\hline 2 & $2.6992 \mathrm{E}-2$ & $4.4159 \mathrm{E}-2$ & $9.2546 \mathrm{E}-3$ & $2.1195 \mathrm{E}-2$ \\
\hline 3 & $6.9896 \mathrm{E}-2$ & $1.4940 \mathrm{E}-2$ & $2.4738 \mathrm{E}-3$ & $6.7478 \mathrm{E}-3$ \\
\hline 4 & $1.7752 \mathrm{E}-3$ & $4.1271 \mathrm{E}-3$ & $6.3125 \mathrm{E}-4$ & $1.9710 \mathrm{E}-3$ \\
\hline 5 & $4.4472 \mathrm{E}-4$ & $1.1075 \mathrm{E}-3$ & $1.6019 \mathrm{E}-4$ & $5.4773 \mathrm{E}-4$ \\
\hline \hline$\alpha$ & 2.3 & 1.78 & 1.95 & 1.76 \\
\hline
\end{tabular}


The superconvergence result in Section 4 is applicable to Example 6.4, which predicts a convergence with $\alpha_{2}=2$ for the velocity. Our numerical experiments reveal a better convergence in this test.

\section{REFERENCES}

[1] I. Babuška, The finite element method with Lagrangian multiplier, Numer. Math., 20 (1973), $179-192$.

[2] F. Brezzi, On the existence, uniqueness, and approximation of saddle point problems arising from Lagrangian multipliers, RAIRO, Anal. Numér., 2 (1974), 129-151.

[3] F. Brezzi, J. Douglas, R. Durán, and L. Marini, Mixed finite elements for second order elliptic problems in three variables, Numer. Math., 52 (1987), 237-250.

[4] F. Brezzi, J. Douglas, M. Fortin, and L. Marini, Efficient rectangular mixed finite elements in two and three spaces variables, RAIRO, Modelisation Math. Anal. Numér., 21 (1987), 581-604.

[5] F. Brezzi, J. Douglas, and L. Marini, Two families of mixed finite elements for second order elliptic problems, Numer. Math., 47 (1985), 217-235.

[6] F. Brezzi and M. Fortin, "Mixed and Hybrid Finite Element Methods", Springer-Verlag, New York, 1991.

[7] P. Ciarlet, Interpolation theory over curved elements, with applications to finite element methods, Comput. Meth. Mech. \& Engin., 1 (1972), 217-249.

[8] J. Douglas and J. Robert, Global estimate for mixed finite elements methods for second order elliptic equations, Math. Comput., 44 (1985), 39-52.

[9] J. Douglas and J Wang, Superconvergence of mixed finite element spaces on rectangular domains, Calcolo, 26 (1989), 121-134.

[10] J. Douglas and J. Wang, A new family of spaces in mixed finite element methods for rectangular elements, Comp. Appl. math. 12 (1993), 183-197.

[11] R. Durán, Error analysis in $L^{p}, 1 \leq p \leq \infty$, for mixed finite element methods for linear and quasi-linear elliptic problems, RAIRO, Anal. Mumér., 22 (1988), 371-387.

[12] R. E. Ewing, R. Lazarov, and J. Wang, Superconvergence of the velocity along the Gauss lines in mixed finite element methods, SIAM J. Numer. Anal., 28 (1991), 1015-1029.

[13] Q. Lin, N. Yan, and A. Zhou, A rectangular test for interpolated finite elements, Proc. Syst. Sci. Syst. Eng., 1991.

[14] Q. Lin and M. Liu, A rectangular test for biharmonic problems, Proc. Syst. Sci. Syst. Eng., 1991.

[15] P. Raviart and J. Thomas, A mixed finite element method for 2nd order elliptic problems, In the "Mathematics Aspects of Finite Ellement methods", Lecture Notes on Mathematics, Vol 606, 292-315, 1977.

[16] J. Wang, Asymptotic expansions and $L^{\infty}$-error estimates for mixed finite element methods for second order elliptic problems, Numer. Math., 55 (1989), 401-430.

[17] J. Wang, Superconvergence and extrapolation for mixed finite element methods on rectangular domains, Math. Comput., 56 (1991), 477-503.

[18] J. Wang and T. Mathew, Mixed finite element method over quadrilaterals, In the Proceedings of the Third International Conference on Advances in Numerical Methods and Applications, Eds: I. T. Dimov, Bl. Sendov, and P. Vassilevski, World Scientific, 1994, 203-214.

Institute for Scientific Computation, Texas A\&M University, College Station, TX 77843

Department of Mathematics, University of Wyoming, Laramie, Wyoming, 82071

Department of Mathematics, University of Wyoming, Laramie, Wyoming, 82071 